OPEN ACCESS

Edited by: Nihal Ahmad, University of Wisconsin-Madison, United States

Reviewed by:

Tapas Ranjan Behera, Cleveland Clinic, United States

Gagan Chhabra,

University of Wisconsin-Madison,

United States

*Correspondence:

Qiwen Zhu

qwenzhu18@163.com

Rong Zhang

zhangrong@syphu.edu.cn

Specialty section:

This article was submitted to Skin Cancer,

a section of the journa

Frontiers in Oncology

Received: 22 September 2021 Accepted: 10 November 2021 Published: 06 December 2021

Citation:

Zhao L, Xia W, Zhang Y, Zou P, Zhu Q and Zhang $R$ (2021) Efficacy and Safety of Immune Checkpoint Blockades in the Treatment of Ocular Melanoma: A Systematic

Review and Meta-Analysis.

Front. Oncol. 11:781162. doi: 10.3389/fonc.2021.781162

\section{Efficacy and Safety of Immune Checkpoint Blockades in the Treatment of Ocular Melanoma: A Systematic Review and Meta-Analysis}

\author{
Lu Zhao ${ }^{1,2}$, Wenwen Xia ${ }^{1}$, Yan Zhang ${ }^{3}$, Peng Zou ${ }^{2}$, Qiwen Zhu ${ }^{4 *}$ and Rong Zhang ${ }^{1 *}$ \\ ${ }^{1}$ Department of Biopharmaceutics, School of Life Science and Bio-Pharmaceutics, Shenyang Pharmaceutical University, \\ Shenyang, China, ${ }^{2}$ Department of Biochemistry and Molecular Biology, School of Basic Medical Sciences, Shenyang \\ Medical College, Shenyang, China, ${ }^{3}$ School of Traditional Chinese Medical, Shenyang Medical College, Shenyang, \\ China, ${ }^{4}$ Key Laboratory of Behavioral and Cognitive Neuroscience of Liaoning Province, Shenyang Medical College, \\ Shenyang, China
}

Aim: This meta-analysis aimed to compare the efficacy and safety of immune checkpoint blockade for the treatment of ocular melanoma.

Methods: We searched the PubMed, EMBASE, and Cochrane Library databases up to July 2021. Effect sizes (ESs) and corresponding 95\% confidence intervals (Cls) were used to compare the outcomes. Efficacy outcomes included complete response (CR), partial response $(\mathrm{PR})$, stable disease $(\mathrm{SD})$, progressive disease $(\mathrm{PD})$, objective response rate (ORR), overall survival (OS), progression free survival (PFS). Safety outcomes included adverse events (AEs) and serious adverse event (SAEs).

Results: A total of 16 eligible articles with 848 ocular melanoma patients were included. ICB treatment significantly improved $C R(E S=0.02,95 \% C l: 0.00-0.03, P=0.023), P R$ ( $E S=0.07,95 \% \mathrm{Cl}: 0.05-0.09, P=0.000$ ), SD (ES=0.31, 95\%Cl: 0.17-0.46, $P=0.000), P D$ ( $E S=0.69$, 95\%Cl: 0.61-0.77, $\mathrm{P}=0.000$ ), ORR ( $E S=0.10,95 \% \mathrm{Cl}: 0.04-0.15, \mathrm{P}=0.000$ ), OS ( $E S=9.68,95 \% \mathrm{Cl}: 7.28-12.07, \mathrm{P}=0.000)$ and $\mathrm{PFS}(\mathrm{ES}=2.88,95 \% \mathrm{Cl}$ : 2.69-3.07, $\mathrm{P}=0.000$ ) in patients with ocular melanoma. Moreover, ICB therapies were associated with reduced AEs ( $E S=0.48,95 \% \mathrm{Cl}$ : 0.30-0.67, $\mathrm{P}=0.000)$ and $\mathrm{SAES}(\mathrm{ES}=0.31,95 \% \mathrm{Cl}$ : 0.18-0.45, $P=0.000$ ).

Conclusions: ICB therapy showed good efficacy and safety in treating patients with ocular melanoma.

Keywords: ocular melanoma, immune checkpoint blockade, PD-1, PD-L1, CTLA-4, meta-analysis 


\section{INTRODUCTION}

Uveal melanoma (UM) is the most common primary ocular malignancy in adults. It originates from melanocytes of the iris, ciliary body, and choroid (1-3). In Europe, the incidence of UM is 4-7 cases/million, and is much rarer than cutaneous melanoma. In a study in 2011, the incidence of uveal melanoma in the US was reported to be 5.1 per million (4). Although UM accounts for $<5 \%$ of all cases in the United States; however, it remains to be the most common primary ocular malignancy in adults in the US and accounts for $85-95 \%$ of all cases of ocular melanoma (5).

Many approaches exist to treat primary UM with the aim of reducing tumor growth and even preserving vision in the affected eye. These approaches include local management by globe-preserving therapy such as brachytherapy, radiotherapy, laser, or surgical resection, or enucleation (5). In 2006, a Collaborative Ocular Melanoma Study Group (COMS) demonstrated that UM patients with medium-sized choroidal melanomas treated with either iodine125 brachytherapy or enucleation demonstrated equivalent survival outcomes (6). In the US, the majority of patients with primary uveal melanomas are treated with first-line plaque brachytherapy.

Despite effective local therapies, nearly $40-50 \%$ of UM patients will ultimately develop distant metastasis $(7,8)$. Up to $95 \%$ of cases of metastatic UM spread to the liver $(2,9,10)$. The median survival of UM patients who have developed liver metastasis is $6-12$ months (11-13).

In recent years, treatment with immune checkpoint blockade (ICB) with programmed death 1 (PD-1) inhibitors as well as ipilimumab, which is an anti-cytotoxic T lymphocyte-associated antigen (CTLA-4) antibody, have yielded promising results for the treatment of cutaneous melanoma (CM) (14-17). However, thus far, no pivotal trials have investigated the efficacy of these treatments in UM.

Currently, immune checkpoint blockade (ICB), which has been successfully used in cutaneous melanoma, is increasingly being adopted for treating UM. In recent years, blocking immune checkpoint proteins such as PD-1/PD-L1 and CTLA-4 have emerged as a pivotal treatment for melanoma patients, which is associated with strong survival benefits $(14,15,18)$. Previous meta-analyses have shown that UM is less responsive to ipilimumab therapy regardless of dosage (19). Therefore, the usefulness of ICB in ocular melanoma requires additional investigation. In the present meta-analysis, we evaluated the efficacy and safety of ICB for the treatment of ocular melanoma.

\section{MATERIALS AND METHODS}

We performed a meta-analysis in accordance with the Preferred Reporting Items for Systematic Reviews and Meta-Analyses (PRISMA2020) guidelines. We started by searching relevant articles by the patients, intervention, comparator, outcomes, and study design (PICOS) principle, and the articles were then screened for inclusion and exclusion criteria.

\section{Eligibility Criteria}

The inclusion criteria included the following: 1) patients who were diagnosed as having ocular melanoma; 2) who received ICB interventions (anti-PD-1/PD-L1 antibodies, anti-CTLA-4 antibodies, PD-1 inhibitors); 3) had efficacious outcomes including complete response (CR), partial response (PR), stable disease $(\mathrm{SD})$, progressive disease $(\mathrm{PD})$, objective response rate (ORR), overall survival (OS), progression-free survival (PFS), and disease control rate (DCR); safety outcomes including adverse events (AEs), serious adverse event (SAEs); 4) study types including cohort and single-arm studies.

The exclusion criteria were as follows: 1) studies that were in the form of conference abstracts, case reports, meta-analysis or review, animal study, and protocol; 2) those that had been written in languages other than English; and 3) studies whose full text could not be obtained or unavailable data.

\section{Search Strategy}

We started a systematic search on the PubMed, EMBASE, and Cochrane Library databases from inception to July 2021 for potentially eligible studies, using the MeSH terms 'ocular melanoma' and 'immune checkpoint inhibitors' and relevant key words.

\section{Data Extraction and Quality Assessment}

The selection and inclusion of studies were performed in two stages by two independent reviewers (LZ and WX). This included the analysis of titles/abstracts followed by the full texts. Disagreements were resolved by a third reviewer (RZ).

The data retrieved included the names of authors, publication year, study design, country; patient characteristics (number of patients, age, gender, sample size, intervention, dosage, follow-up time, and outcomes).

The methodological quality of the cohort and single-arm studies was evaluated using the Newcastle and Ottawa Scale (NOS) (20), with the maximum possible score of 9 points representing the least risk of bias. Quality assessment was performed in duplicate by 2 researchers separately (LZ and WX).

\section{Statistical Analysis}

Effect sizes (ESs) and corresponding 95\% confidence intervals (CIs) were used to compare the outcomes. Cochran's $\mathrm{Q}$ statistic $(\mathrm{P}<0.10$ indicated evidence of heterogeneity) assessed the heterogeneity among studies (21). When significant heterogeneity $(\mathrm{P}<0.10)$ was detected, the random-effects model was used to combine the effect sizes of the included studies. Otherwise, the fixed-effects model was adopted. (Higgins, J.P.T. and Green, S. (2011) Cochrane handbook for systematic reviews of interventions version 5.1.0. Naunyn Schmied. Arch. Exp. Pathol. Pharmakol. 5, S38) (22). All analyses were performed using STATA SE software version 14.0 (StataCorp, College Station, Texas, USA).

\section{RESULTS}

\section{Identification of Eligible Studies}

A total of 769 relevant publications were initially identified via databases and registers. Duplicates and other ineligible records defined by automation tools were removed. The remaining 318 publications were screened for eligibility. Due to insufficient data 
information, non-human research, and language restriction, 302 documents were further excluded. Finally, 16 full-text articles were included for this meta-analysis. Figure $\mathbf{1}$ is a flowchart illustrating the screening process.

\section{Study Characteristics and Quality Assessment}

Table 1 describes the characteristics of the 16 eligible articles (2338) consisting of 13 cohort and 3 single-arm studies. A total of 848 ocular melanoma patients were enrolled, with an age range of 16-65 years. The proportion of male patients ranged from $29.4 \%$ to $63.6 \%$. Interventions included anti-PD-1-antibodies (nivolumab, pembrolizumab), anti-CTLA-4 antibodies (ipilimumab, tremelimumab), anti-PD-L1 antibodies, and PD-1 inhibitor. The follow-up time ranged from 12-96 weeks. The treatment outcomes included complete response (CR), partial response (PR), stable disease (SD), progressive disease (PD) and objective response rate (ORR), overall survival (OS), and progression-free survival (PFS).

\section{Identification of studies via databases and registers}

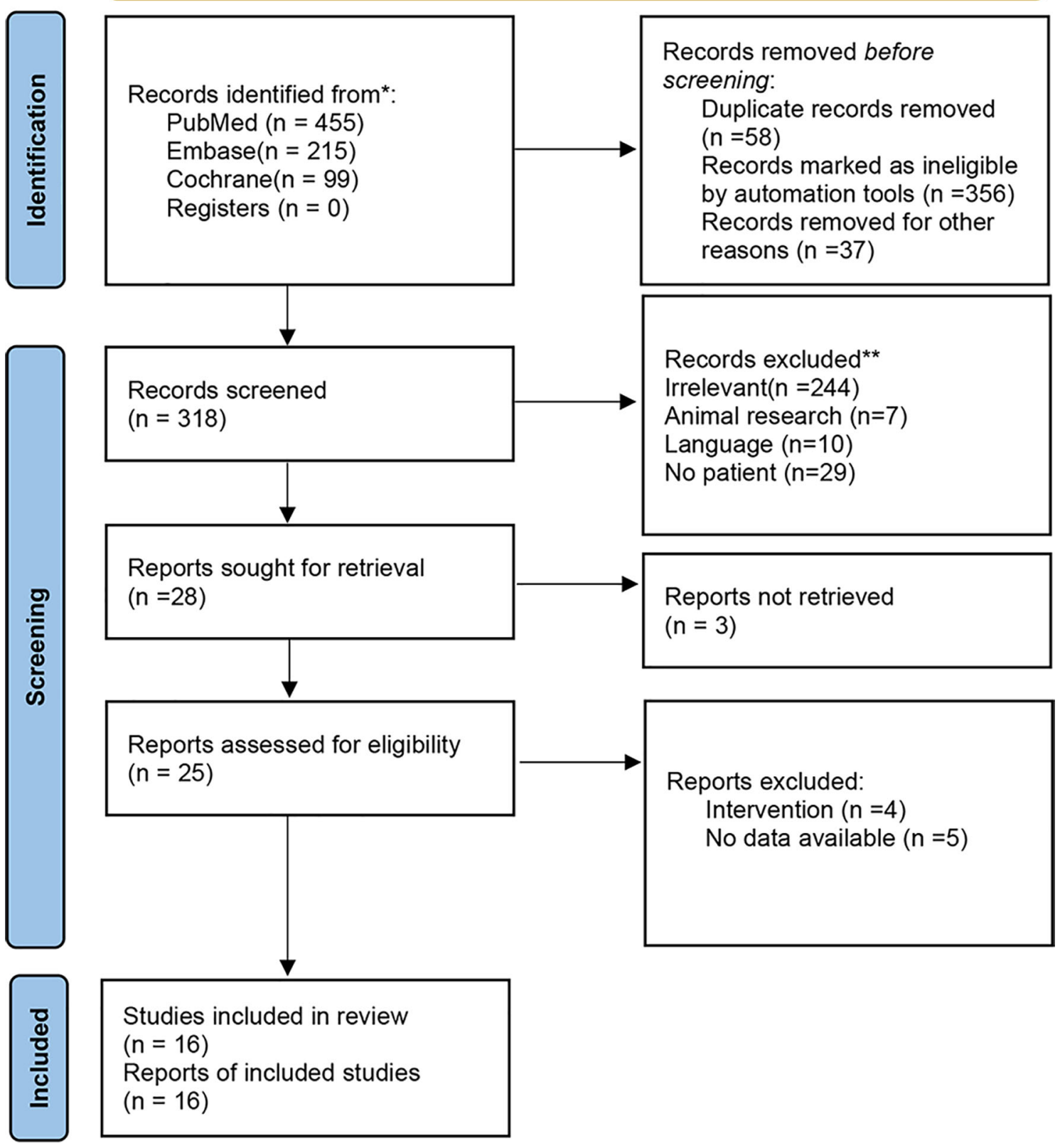

FIGURE 1 | Consort chart of the included/excluded studies. 


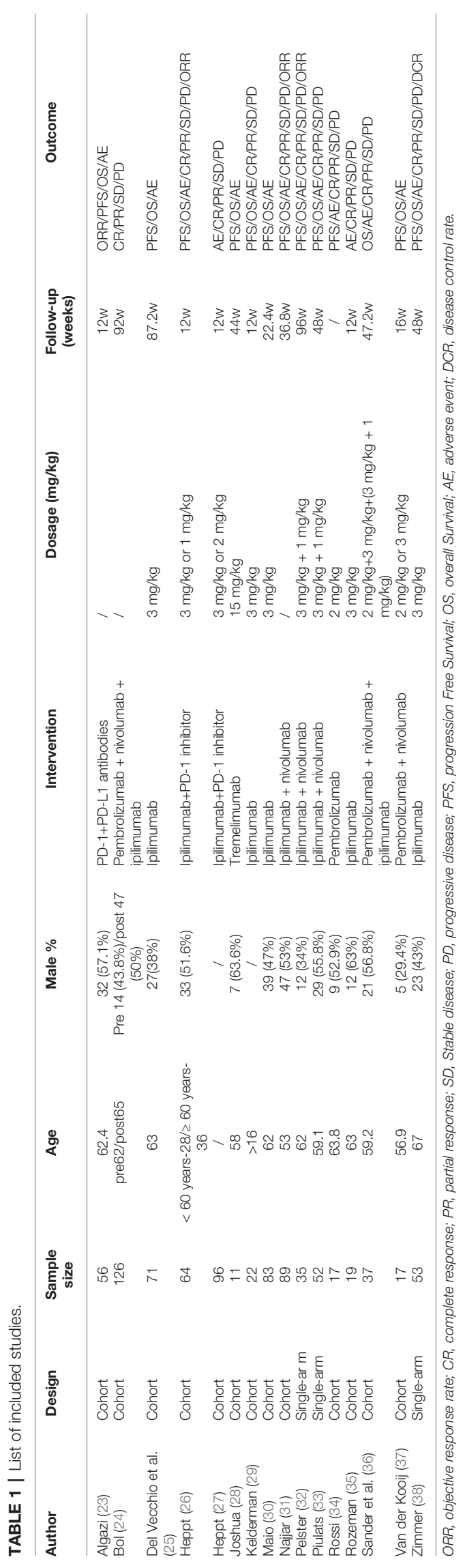

NOS analysis deemed all 13 cohort studies to be of high methodological quality. Two single-arm studies scored 12, and one scored 13 according to the Methodological Index for NonRandomized Studies (MINORS), indicating medium quality (Table 2).

\section{CR, PR, SD, PD, and ORR Outcomes}

Six studies reported the outcome of CR in ICB treatment for ocular melanoma. The pooled results suggested that immune checkpoint blockage significantly improved CR in patients with ocular melanoma (ES $=0.02,95 \%$ CI: $0.00-0.03, \mathrm{P}=0.023 ; \mathrm{I}^{2}=$ $0.0 \%, \mathrm{P}_{\text {heterogeneity }}=0.900$ ) (Figure $\mathbf{2 A}$ ). Subgroup analysis showed no significant increase in the $\mathrm{CR}$ between ipilimumab ( $\mathrm{ES}=0.01,95 \%$ CI: $-0.01-0.04, \mathrm{P}=0.284 ; \mathrm{I}^{2}=0.0 \%, \mathrm{P}_{\text {heterogeneity }}=$ 0.445 ), ipilimumab plus PD-1 inhibitor (ES $=0.03,95 \%$ CI: -0.01 $0.07, \mathrm{P}=0.152$ ); , and ipilimumab plus nivolumab ( $\mathrm{ES}=0.01,95 \%$ CI: $\left.-0.00-0.03, \mathrm{P}=0.108 ; \mathrm{I}^{2}=0.0 \%, \mathrm{P}_{\text {heterogeneity }}=0.807\right)$. No heterogeneity was detected between groups $\left(\mathrm{P}_{\text {heterogeneity }}=0.743\right.$, Figure S1A).

Twelve studies reported PR results. Pooled analysis suggested that the immune interventions significantly improved the PR $\left(\mathrm{ES}=0.07,95 \%\right.$ CI: $0.05-0.09, \mathrm{P}=0.000 ; \mathrm{I}^{2}=0.0 \%, \mathrm{P}_{\text {heterogeneity }}=$ 0.622) (Figure 2B). Subgroup analysis showed significant benefits in the PR for treatments using pembrolizumab plus nivolumab and pilimumab ( $\mathrm{ES}=0.06,95 \% \mathrm{CI}$ : $0.02-0.09, \mathrm{P}=$ $\left.0.002 ; \mathrm{I}^{2}=0.0 \%, \mathrm{P}_{\text {heterogeneity }}=0.907\right)$, ipilimumab $(\mathrm{ES}=0.08$, 95\%CI: $0.02-0.13, \mathrm{P}=0.005 ; \mathrm{I}^{2}=0.0 \%, \mathrm{P}_{\text {heterogeneity }}=0.335$ ), ipilimumab plus PD-1 inhibitor (ES $=0.13,95 \%$ CI: $0.05-0.21$, $\left.\mathrm{P}=0.001 ; \mathrm{I}^{2}=0.0 \%, \mathrm{P}_{\text {heterogeneity }}=0.716\right)$, pembrolizumab $(\mathrm{ES}=$ $0.07,95 \%$ CI: $0.01-0.12, \mathrm{P}=0.026 ; \mathrm{I}^{2}=0.0 \%, \mathrm{P}_{\text {heterogeneity }}=$ 0.476 ), and ipilimumab plus nivolumab ( $\mathrm{ES}=0.11,95 \% \mathrm{CI}$ : $\left.0.06-0.15, \mathrm{P}=0.000 ; \mathrm{I}^{2}=0.0 \%, \mathrm{P}_{\text {heterogeneity }}=0.742\right)$. The use of nivolumab alone did not increase the PR (ES $=0.03,95 \%$ CI: $0.03-0.09, \mathrm{P}=0.312$ ). The heterogeneity across different groups was not significant $\left(\mathrm{P}_{\text {heterogeneity }}=0.235\right.$, Figure S1B $)$.

Fourteen studies reported the data of SD. Overall, significant improvement in SD was observed for ocular melanoma (ES = $0.31,95 \%$ CI: $0.17-0.46, \mathrm{P}=0.000 ; \mathrm{I}^{2}=95.1 \%, \mathrm{P}_{\text {heterogeneity }}=$ 0.000 ) (Figure 2C). The SD was also found to increase when using various immune checkpoint inhibitors including pembrolizumab with nivolumab and ipilimumab $(\mathrm{ES}=0.20$, 95\% CI: $0.14-0.27, \mathrm{P}=0.000 ; \mathrm{I}^{2}=6.3 \%, \mathrm{P}_{\text {heterogeneity }}=0.302$ ), ipilimumab ( $\mathrm{ES}=0.45,95 \%$ CI: $0.02-0.87, \mathrm{P}=0.038 ; \mathrm{I}^{2}=98.1 \%$, $\left.\mathrm{P}_{\text {heterogeneity }}=0.000\right)$, ipilimumab plus $\mathrm{PD}-1$ inhibitor $(\mathrm{ES}=0.21$, 95\% CI: $0.12-0.30, \mathrm{P}=0.000 ; \mathrm{I}^{2}=0.0 \%, \mathrm{P}_{\text {heterogeneity }}=0.663$ ), pembrolizumab $\left(\mathrm{ES}=0.23,95 \% \mathrm{CI}: 0.06-0.40, \mathrm{P}=0.007 ; \mathrm{I}^{2}=\right.$ $\left.51.9 \%, \mathrm{P}_{\text {heterogeneity }}=0.149\right)$, nivolumab $(\mathrm{ES}=0.16,95 \% \mathrm{CI}: 0.03$ $0.28, \mathrm{P}=0.015$ ), and ipilimumab plus nivolumab ( $\mathrm{ES}=0.36,95 \%$ CI: $0.19-0.53, \mathrm{P}=0.000 ; \mathrm{I}^{2}=82.4 \%, \mathrm{P}_{\text {heterogeneity }}=0.003$ ) (Figure S1C).

Fourteen studies reported PD outcome. Results of the pooled analysis revealed that treatment with ICB improved the PD (ES = $0.69,95 \%$ CI: $0.61-0.77, \mathrm{P}=0.000 ; \mathrm{I}^{2}=80.2 \%, \mathrm{P}_{\text {heterogeneity }}=$ 0.000 ) (Figure 2D). Similar benefits were shown for the PD when using treatments of pembrolizumab combined with nivolumab and ipilimumab ( $\mathrm{ES}=0.67,95 \%$ CI: $0.49-0.85, \mathrm{P}=0.000 ; \mathrm{I}^{2}=$ $\left.76.3 \%, \mathrm{P}_{\text {heterogeneity }}=0.040\right)$, ipilimumab $(\mathrm{ES}=0.67,95 \% \mathrm{CI}$ : 


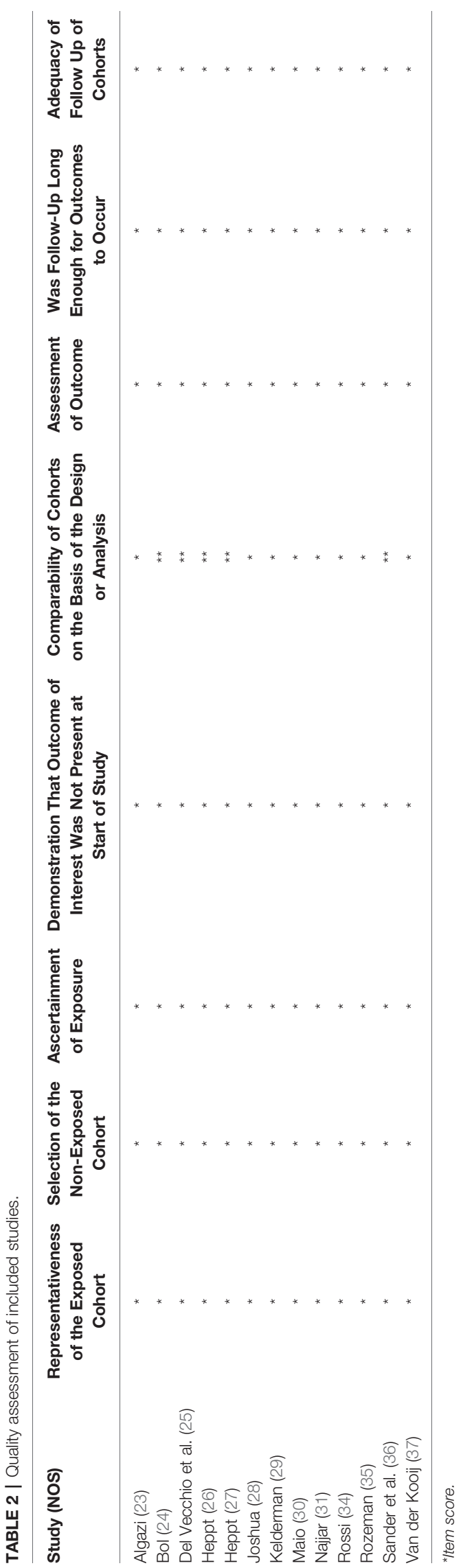

$\left.0.51-0.83, \mathrm{P}=0.000 ; \mathrm{I}^{2}=71.4 \%, \mathrm{P}_{\text {heterogeneity }}=0.030\right)$, ipilimumab plus PD-1 inhibitor (ES = 0.58, 95\% CI: 0.47-0.69, $\left.\mathrm{P}=0.000 ; \mathrm{I}^{2}=0.0 \%, \mathrm{P}_{\text {heterogeneity }}=0.487\right)$, pembrolizumab $(\mathrm{ES}=$ $0.67,95 \%$ CI: $0.45-0.88, \mathrm{P}=0.000 ; \mathrm{I}^{2}=64.2 \%, \mathrm{P}_{\text {heterogeneity }}=$ 0.095), nivolumab ( $\mathrm{ES}=0.89,95 \% \mathrm{CI}: 0.76-1.03, \mathrm{P}=0.000 ; \mathrm{I}^{2}=$ $66.8 \%, \mathrm{P}_{\text {heterogeneity }}=0.083$ ), and ipilimumab combined with nivolumab ( $\mathrm{ES}=0.65,95 \% \mathrm{CI}$ : $0.48-0.82, \mathrm{P}=0.000 ; \mathrm{I}^{2}=82.7 \%$, $\left.\mathrm{P}_{\text {heterogeneity }}=0.003\right)($ Figure S1D).

Pooled analysis of five studies suggested beneficial ORR outcome ( $E S=0.10,95 \%$ CI: $0.04-0.15, P=0.000 ; \mathrm{I}^{2}=58.9 \%$, $\left.\mathrm{P}_{\text {heterogeneity }}=0.045\right)($ Figure $2 \mathrm{E}$ ). The ORR was also found to increase when using ipilimumab plus $\mathrm{PD}-1$ inhibitor $(\mathrm{ES}=0.16$, 95\% CI: $0.07-0.24, \mathrm{P}=0.000)$ and ipilimumab combined with nivolumab ( $\mathrm{ES}=0.13,95 \% \mathrm{CI}: 0.07-0.19, \mathrm{P}=0.000 ; \mathrm{I}^{2}=0.0 \%$, $\left.\mathrm{P}_{\text {heterogeneity }}=0.395\right)$. However, no improvements were seen in the ORR when using pembrolizumab in combination with nivolumab and ipilimumab $(\mathrm{ES}=0.05,95 \% \mathrm{CI}$ : $-0.02-0.13, \mathrm{P}=$ $0.146), \mathrm{PD}-1$, and PD-L1 antibodies (ES $=0.04,95 \% \mathrm{CI}:-0.01-$ $0.08, \mathrm{P}=0.148$ ) (Figure S1E).

\section{OS and PFS Outcomes}

Pooled analysis including eleven studies suggested favorable OS outcome $\left(\mathrm{ES}=9.68,95 \% \mathrm{CI}: 7.28-12.07, \mathrm{P}=0.000 ; \mathrm{I}^{2}=81.4 \%\right.$, $\left.\mathrm{P}_{\text {heterogeneity }}=0.000\right)$ (Figure 3A). Subgroup analysis showed similar OS benefits when treatments used PD-1 and PD-L1 antibodies ( $\mathrm{ES}=7.70,95 \% \mathrm{CI}: 0.75-14.65, \mathrm{P}=0.030)$, ipilimumab ( $\mathrm{ES}=6.10,95 \% \mathrm{CI}$ : 5.06-7.15, $\mathrm{P}=0.000 ; \mathrm{I}^{2}=0.0 \%$, $\left.\mathrm{P}_{\text {heterogeneity }}=0.794\right)$, ipilimumab plus $\mathrm{PD}-1$ inhibitor $(\mathrm{ES}=$ 16.10, 95\% CI: $12.90-19.30, \mathrm{P}=0.000)$, tremelimumab (ES = 12.80, 95\% CI: 4.85-20.75, $\mathrm{P}=0.002)$, ipilimumab plus nivolumab ( $E S=13.90$, 95\%CI: 10.03-17.77, $\mathrm{P}=0.000$; $\mathrm{I}^{2}=0.0 \%$, $\left.\mathrm{P}_{\text {heterogeneity }}=0.561\right)$, pembrolizumab plus nivolumab plus ipilimumab ( $E S=15.60,95 \% \mathrm{CI}: 8.80-22.40, \mathrm{P}=0.000)$, and pembrolizumab plus nivolumab ( $\mathrm{ES}=8.97,95 \% \mathrm{CI}$ : $4.58-13.37$, $\mathrm{P}=0.000)$ (Figure S2A).

Pooled results with twelve studies suggested favorable PFS outcome ( $\mathrm{ES}=2.88,95 \% \mathrm{CI}: 2.69-3.07, \mathrm{P}=0.000 ; \mathrm{I}^{2}=58.3 \%$, $\left.\mathrm{P}_{\text {heterogeneity }}=0.006\right)($ Figure 3B). The PFS was found to improve in treatments using PD-1 and PD-L1 antibodies (ES $=2.60,95 \%$ CI: $2.40-2.80, \mathrm{P}=0.000)$, ipilimumab ( $\mathrm{ES}=3.38$, 95\%CI: $2.60-$ $\left.4.16, \mathrm{P}=0.000 ; \mathrm{I}^{2}=77.2 \%, \mathrm{P}_{\text {heterogeneity }}=0.004\right)$, ipilimumab plus PD-1 inhibitor (ES $=3.00,95 \% \mathrm{CI}: 2.40-2.60, \mathrm{P}=0.000)$, tremelimumab ( $\mathrm{ES}=2.90,95 \% \mathrm{CI}: 2.80-3.00, \mathrm{P}=0.000)$, ipilimumab plus nivolumab ( $\mathrm{ES}=2.97,95 \% \mathrm{CI}: 2.20-3.74, \mathrm{P}=$ $\left.0.000 ; \mathrm{I}^{2}=42.6 \%, \mathrm{P}_{\text {heterogeneity }}=0.175\right)$, pembrolizumab in combination with nivolumab and ipilimumab $(\mathrm{ES}=3.80,95 \%$ CI: $0.40-7.20, \mathrm{P}=0.028)$, and pembrolizumab plus nivolumab $(\mathrm{ES}=2.40,95 \% \mathrm{CI}: 1.69-3.11, \mathrm{P}=0.000)$ (Figure S2B).

\section{Safety Outcomes}

Pooled analysis of fourteen studies suggested significantly reduced AEs after treatment with immune checkpoint blockade ( $\mathrm{ES}=0.48,95 \% \mathrm{CI}: 0.30-0.67, \mathrm{P}=0.000$; $\mathrm{I}^{2}=97.2 \%$, $\left.\mathrm{P}_{\text {heterogeneity }}=0.000\right)($ Figure 4A). The AEs also decreased when using PD-1 and PD-L1 antibodies (ES = 0.13, 95\% CI: 0.04-0.21, $\mathrm{P}=0.000$ ), ipilimumab ( $\mathrm{ES}=0.60,95 \% \mathrm{CI}: 0.31-0.88, \mathrm{P}=0.000$; $\left.\mathrm{I}^{2}=96.1 \%, \mathrm{P}_{\text {heterogeneity }}=0.000\right)$, ipilimumab plus PD-1 inhibitor 


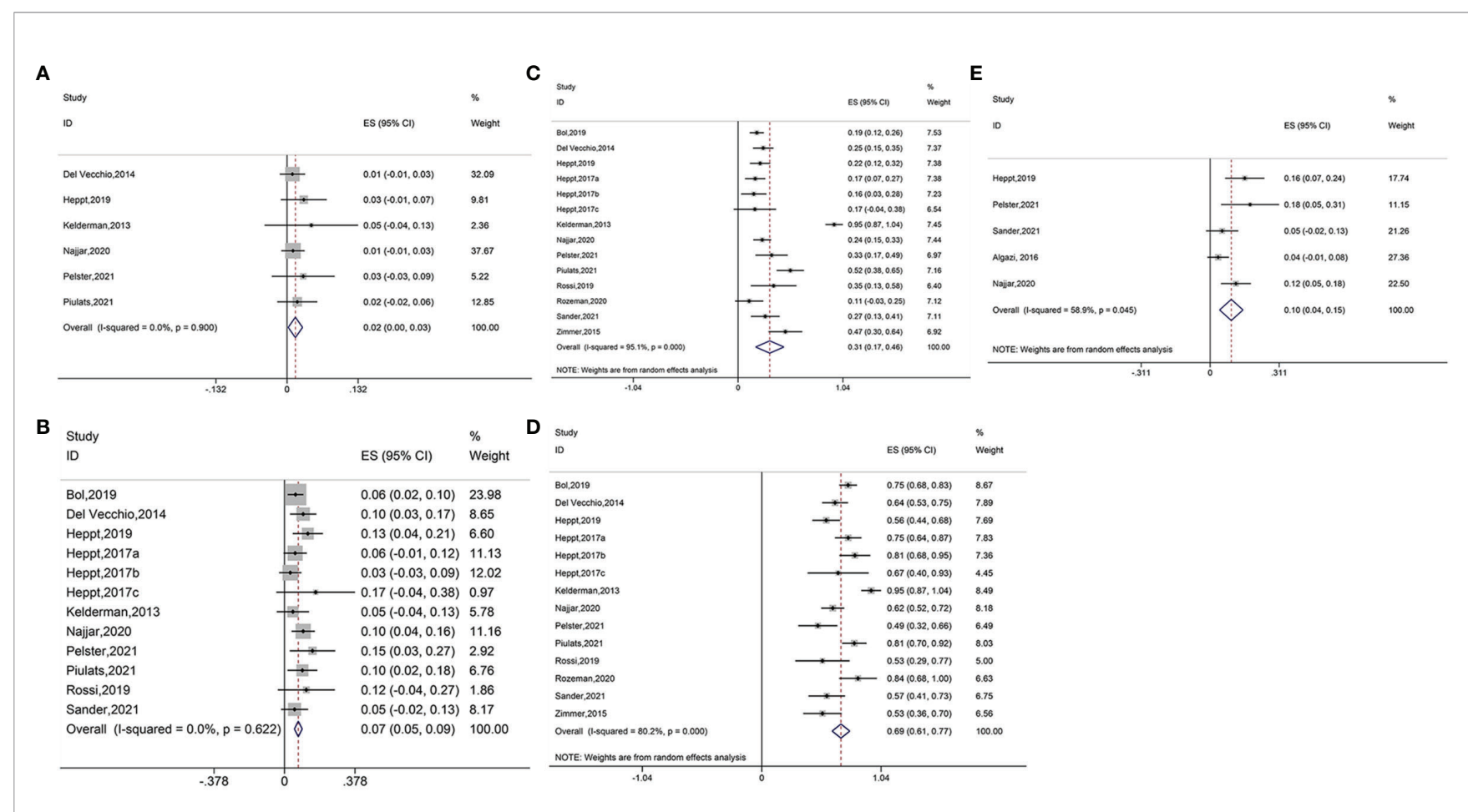

FIGURE 2 | (A) Forest plot of complete response (CR) with immune checkpoint blockade treatment. (B) Forest plot of partial response (PR) with immune checkpoint blockade treatment. (C) Forest plot of stable disease (SD) with immune checkpoint blockade treatment. (D) Forest plot of progressive disease (PD) with immune checkpoint blockade treatment. (E) Forest plot of objective response rate (ORR) with immune checkpoint blockade treatment.

$\left(\mathrm{ES}=0.58,95 \%\right.$ CI: $0.47-0.69, \mathrm{P}=0.000 ; \mathrm{I}^{2}=0.0 \%, \mathrm{P}_{\text {heterogeneity }}=$ $0.319)$, pembrolizumab ( $\mathrm{ES}=0.23,95 \% \mathrm{CI}: 0.14-0.33, \mathrm{P}=0.000$; $\left.\mathrm{I}^{2}=0.0 \%, \mathrm{P}_{\text {heterogeneity }}=0.450\right)$, nivolumab $(\mathrm{ES}=0.41,95 \% \mathrm{CI}$ : $0.24-0.58, \mathrm{P}=0.000$ ); ipilimumab plus nivolumab $(\mathrm{ES}=0.90$, 95\% CI: $0.79-1.01, \mathrm{P}=0.000 ; \mathrm{I}^{2}=59.5 \%, \mathrm{P}_{\text {heterogeneity }}=0.116$ ), and pembrolizumab in combination with nivolumab and ipilimumab ( $\mathrm{ES}=0.51,95 \% \mathrm{CI}: 0.35-0.68, \mathrm{P}=0.000)$. The AEs did not significantly reduce when using pembrolizumab plus nivolumab ( $\mathrm{ES}=0.06,95 \% \mathrm{CI}:-0.05-0.17, \mathrm{P}=0.302$ ) (Figure S3A). Furthermore, all treatment modalities investigated were associated with significantly reduced AEs and SAEs.

Pooled analysis of ten studies suggested significantly reduced SAEs after immune checkpoint blockade treatments $(E S=0.31,95 \%$ CI: $0.18-0.45, \mathrm{P}=0.000 ; \mathrm{I}^{2}=92.8 \%, \mathrm{P}_{\text {heterogeneity }}=0.000$ )

(Figure 4B). Subgroup analysis showed decreased SAEs when using ipilimumab (ES = 0.17, 95\% CI: 0.07-0.28, P = 0.001; $\mathrm{I}^{2}=$ $\left.82.1 \%, \mathrm{P}_{\text {heterogeneity }}=0.000\right)$, ipilimumab plus $\mathrm{PD}-1$ inhibitor $(\mathrm{ES}=$ 0.58, 95\% CI: 0.46-0.70, P = 0.000), tremelimumab $(\mathrm{ES}=0.55,95 \%$ CI: $0.25-0.84, \mathrm{P}=0.000$ ), ipilimumab plus nivolumab ( $\mathrm{ES}=0.49$, 95\% CI: $\left.0.32-0.67, \mathrm{P}=0.000 ; \mathrm{I}^{2}=63.1 \%, \mathrm{P}_{\text {heterogeneity }}=0.100\right)$, and pembrolizumab in combination with nivolumab and ipilimumab $(\mathrm{ES}=0.19,95 \%$ CI: 0.06-0.32, $\mathrm{P}=0.003)$ (Figure S3B).

\section{Sensitivity Analysis}

Sensitivity analysis showed that certain individual study exclusions could influence the overall estimated effects of AE, $\mathrm{PD}, \mathrm{SAE}$, and SD, without affecting the OS, PFS, and PR (Figure S6).

\section{Publication Bias}

The Begg test for funnel plot asymmetry indicated the presence of significant publication bias for $\mathrm{PR}(\mathrm{P}=0.043)$ and SAE $(\mathrm{P}=$ 0.015). No significant publication bias was detected for the $\mathrm{AE}$ $(\mathrm{P}=0.190), \mathrm{OS}(\mathrm{P}=0.551), \mathrm{PD}(\mathrm{P}=0.077), \mathrm{PFS}(\mathrm{P}=0.250)$, and $\mathrm{SD}(\mathrm{P}=0.824$; Figure S5).

\section{DISCUSSION}

This meta-analysis systematically evaluated the efficacy and safety of immune checkpoint blockade in the treatment of patients with ocular melanoma. The results showed that immune checkpoint blockade significantly improved the outcomes of CR, PR, SD, PD, ORR, OS, and PFS in ocular melanoma patients. In addition, immune checkpoint blockade was associated with reduced AEs and SAEs.

This meta-analysis included cohort and phase-II single-arm studies investigating the effect of ICB treatment for ocular melanoma. Immune-related interventions covered different immune checkpoint inhibitors. Six studies investigated CTLA4 blockade monotherapy with ipilimumab (at $3 \mathrm{mg} / \mathrm{kg}$ ) or tremelimumab (at $15 \mathrm{mg} / \mathrm{kg}$ ), and one study investigated PD-1 blockade monotherapy (pembrolizumab at $2 \mathrm{mg} / \mathrm{kg}$ ). Additionally, seven studies investigated the combination of CTLA4 and PD-1 blockade, including PD-1 inhibitors or antiPD-1 antibodies. Combined anti-PD-1 antibodies including 


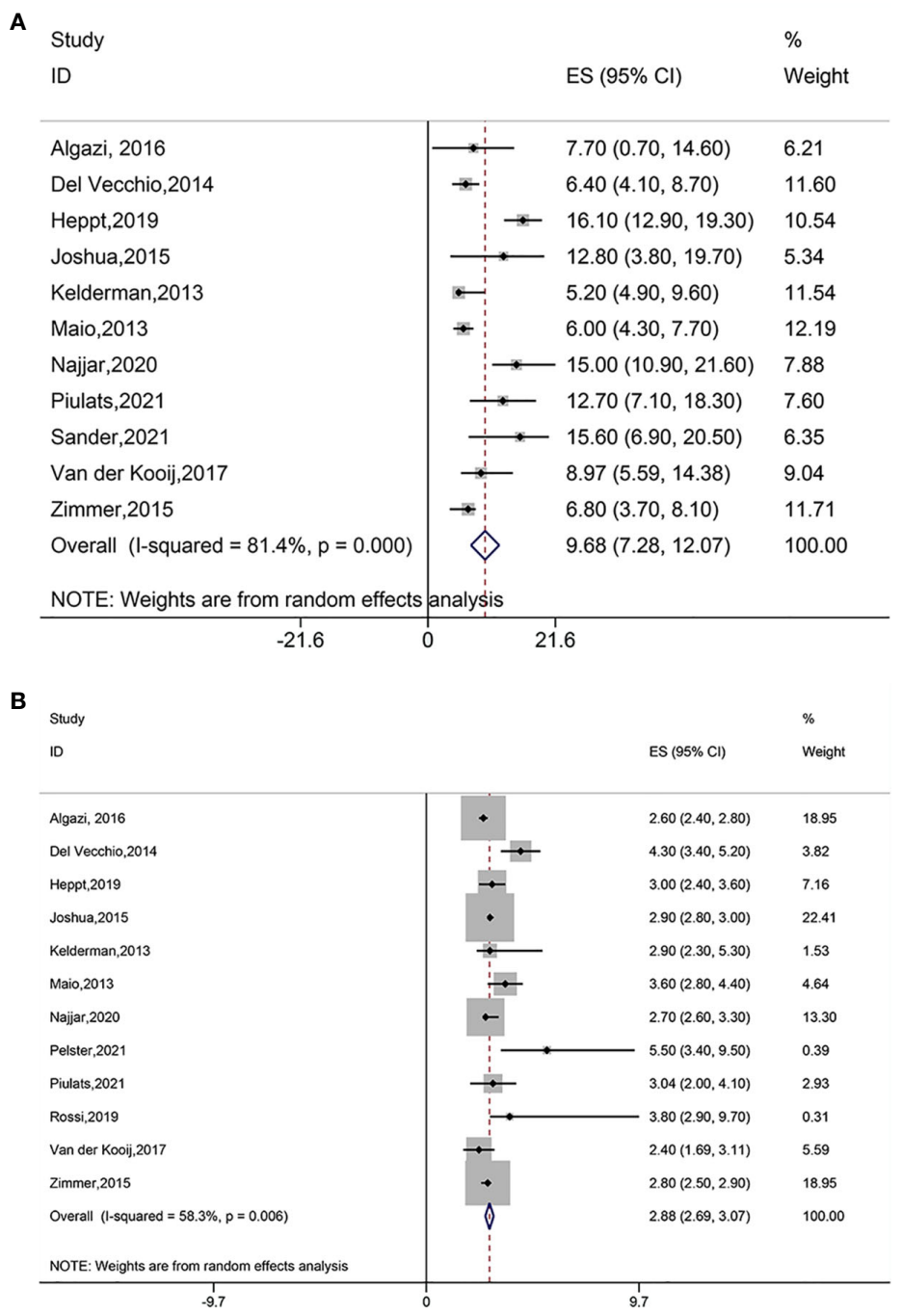

FIGURE 3 | (A) Forest plot of overall survival (OS) of immune checkpoint blockade treatment. (B) Forest plot of progression free survival (PFS) of immune checkpoint blockade treatment.

pembrolizumab and nivolumab, and anti-PD-1 plus anti-PD-L1 antibodies were also evaluated in one study, respectively. This meta-analysis showed that all treatment modalities significantly improved the SD, PD, OS, and PFS of patients regardless of immune checkpoint inhibitor types, indicating stable disease and long-term survival benefits. Subgroup analysis revealed that CTLA-4 blockade monotherapy (ipilimumab), combined CTLA-4 and PD-1 blockade (ipilimumab with Nivolumab or PD-1 inhibitor) did not improve the CR of patients. The monotherapy with anti-PD-1 antibody nivolumab did not increase PR. Additionally, combined PD-1 and CTLA-4 blockade using pembrolizumab, nivolumab and ipilimumab did not lead to an ORR benefit. These results suggested that, compared with monotherapy, the combination of CTLA-4 and PD-1 blockade might be more effective on the clinical response of $\mathrm{PR}$, but not on the CR and ORR for ocular melanoma patients.
Furthermore, immune checkpoint inhibitor therapies were associated with significantly reduced AEs and SAEs, suggesting a good safety profile for 4ICB treatment.

A previous review by Heppt et al. suggested that ICB had lower efficacy in the treatment of advanced uveal melanoma (UM) patients and maintained a high toxicity profile (19). The results showed that UM patients exhibited a minimal response to CTLA-4 blockade monotherapy (ipilimumab or tremelimumab) in terms of the ORR, PFS, and OS. Anti-PD-1 antibodies including pembrolizumab $(2 \mathrm{mg} / \mathrm{kg})$ and nivolumab $(3 \mathrm{mg} / \mathrm{kg})$ yielded an ORR of $30 \%$ and $6 \%$, respectively. No treatment response was observed with CTLA-4 and PD-1 combined blockade, and the median PFS was 2.9 months. Their study comprised of 12 records from 7 expanded access programs (EAP) or named patient programs (NPP), 4 phase II trials, and 1 phase Ib trial for a qualitative synthesis, and no RCT was 


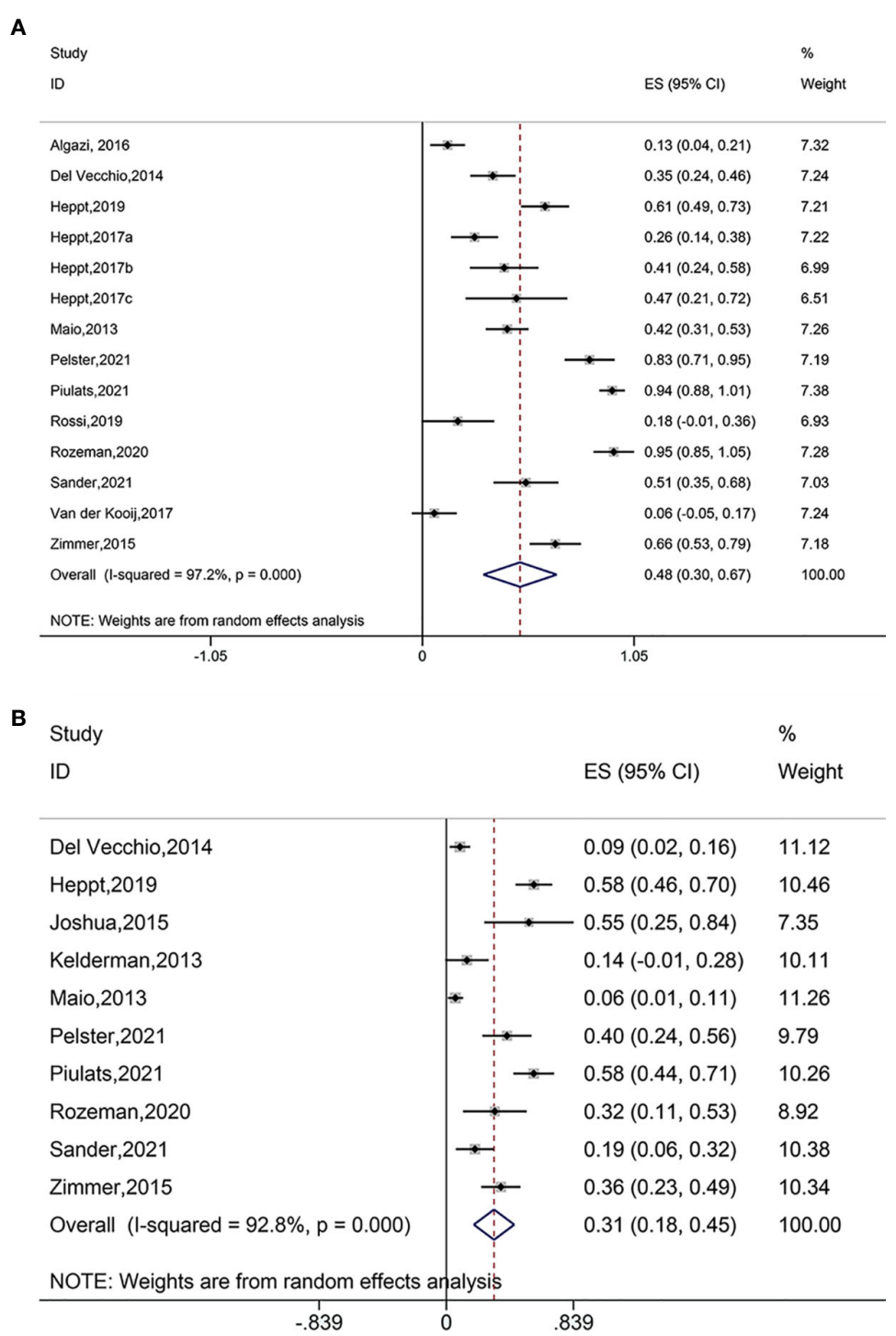

FIGURE 4 | (A) Forest plot of adverse events (AEs) after treatment with immune checkpoint blockade. (B) Forest plot of serious adverse event (SAEs) after treatment with immune checkpoint blockade.

found. Conference abstracts with incomplete or preliminary data were also included. Therefore, there was a high selection bias in that review. The effects of mixed response on outcomes were reported but could not be combined for a quantitative analysis.

Our meta-analysis also demonstrated the favorable outcome of disease control rate (DCR) following ICB treatment (Figure S4). Vecchio et al. reported immune-related disease control for $36 \%$ of patients with metastatic mucosal melanoma, and in most cases, the improved DCR was attributed to SD rather than to a significant reduction in the tumor burden (36). Sander et al. (36) showed that beneficial ORR was notable for metastatic UM patients who achieved a PR, and patients having attained either a PR or SD had an improved DCR (36). Moreover, though a modified immune prognostic index (IPI) status might be used to predict a survival benefit from ICB treatment, the IPI score was not found to be associated with ORR and DCR. The most likely explanation was that the statistical power was sufficient for OS analysis, rather than ORR and DCR assessments. Favorable ORR (defined as CR +PR) and DCR (defined as $\mathrm{SD}+\mathrm{CR}+\mathrm{PR}$ ) with ICB in treating metastatic UM have also been reported $(38,39)$.

The present study has several noted limitations. First, due to the retrospective and uncontrolled design, the results should be interpreted with caution. Secondly, some of the included studies had a relatively small sample size, which would produce an overestimate of the treatment effect when compared with larger studies. Third, no RCTs were identified in this study. The current evidence supporting the application of ICB in UM still needs to be verified in the more rigorously designed RCTs. 


\section{CONCLUSION}

This meta-analysis showed that ICB was effective in treating ocular melanoma patients in terms of patients' clinical responses and long-term survival, with a good safety profile. Further largerscale more rigorously studies are warranted to confirm our findings.

\section{DATA AVAILABILITY STATEMENT}

The original contributions presented in the study are included in the article/Supplementary Material. Further inquiries can be directed to the corresponding authors.

\section{AUTHOR CONTRIBUTIONS}

LZ: Methodology, Formal Analysis, Resources, Writing Original Draft Preparation, Writing Review and Editing. WX: Software, Data Curation. YZ: Conceptualization. PZ: Investigation. QZ: Validation, Visualization, Supervision. RZ: Project

\section{REFERENCES}

1. Jager MJ, Shields CL, Cebulla CM, Abdel-Rahman MH, Grossniklaus HE, Stern MH, et al. Uveal Melanoma. Nat Rev Dis Primers (2020) 6:24. doi: 10.1038/s41572-020-0158-0

2. Carvajal RD, Schwartz GK, Tezel T, Marr B, Francis JH, Nathan PD. Metastatic Disease From Uveal Melanoma: Treatment Options and Future Prospects. Br J Ophthalmol (2017) 101:38-44. doi: 10.1136/bjophthalmol-2016-309034

3. Seider MI, Mruthyunjaya P. Molecular Prognostics for Uveal Melanoma. Retina (2018) 38:211-9. doi: 10.1097/IAE.0000000000001757

4. Mallone S, De Vries E, Guzzo M, Midena E, Verne J, Coebergh JW, et al. Descriptive Epidemiology of Malignant Mucosal and Uveal Melanomas and Adnexal Skin Carcinomas in Europe. Eur J Cancer (Oxford Engl 1990) (2012) 48:1167-75. doi: 10.1016/j.ejca.2011.10.004

5. Yang J, Manson DK, Marr BP, Carvajal RD. Treatment of Uveal Melanoma: Where are We Now? Ther Adv Med Oncol (2018) 10:1758834018757175. doi: $10.1177 / 1758834018757175$

6. Collaborative Ocular Melanoma Study Group. The COMS Randomized Trial of Iodine 125 Brachytherapy for Choroidal Melanoma: V. Twelve-Year Mortality Rates and Prognostic Factors: COMS Report No. 28. Arch Ophthalmol (Chicago Ill 1960) (2006) 124:1684-93. doi: 10.1001/archopht.124.12.1684

7. Pelster MS, Gruschkus SK, Bassett R, Gombos DS, Shephard M, Posada L, et al. Nivolumab and Ipilimumab in Metastatic Uveal Melanoma: Results From a Single-Arm Phase II Study. J Clin Oncol Off J Am Soc Clin Oncol (2021) 39:599-607. doi: 10.1200/jco.20.00605

8. Krantz BA, Dave N, Komatsubara KM, Marr BP, Carvajal RD. Uveal Melanoma: Epidemiology, Etiology, and Treatment of Primary Disease. Clin Ophthalmol (2017) 11:279-89. doi: 10.2147/OPTH.S89591

9. Kaliki S, Shields CL. Uveal Melanoma: Relatively Rare But Deadly Cancer. Eye (Lond) (2017) 31:241-57. doi: 10.1038/eye.2016.275

10. Bellerive C, Ouellet E, Kamaya A, Singh AD. Liver Imaging Techniques: Recognition of Uveal Melanoma Metastases. Ocul Oncol Pathol (2018) 4:25460. doi: 10.1159/000485424

11. Kolandjian NA, Wei C, Patel SP, Richard JL, Dett T, Papadopoulos NE, et al. Delayed Systemic Recurrence of Uveal Melanoma. Am J Clin Oncol (2013) 36:443-9. doi: 10.1097/COC.0b013e3182546a6b

12. Rietschel P, Panageas KS, Hanlon C, Patel A, Abramson DH, Chapman PB. Variates of Survival in Metastatic Uveal Melanoma. J Clin Oncol (2005) 23:8076-80. doi: 10.1200/JCO.2005.02.6534
Administration, Funding Acquisition. All authors contributed to the article and approved the submitted version.

\section{FUNDING}

This work was supported by the National Natural Science Foundation of China [Grant numbers: 31772518]; Innovation Team and Talent Support Programs of Colleges in Liaoning Province [Grant numbers: LT2019013 and LR2019071]; Support Plan of Shenyang Pharmaceutical University for Youth Development [Grant Number: ZQN2014A05]; Natural Science Foundation of Liaoning Province [Grant Number: 2019MS-295].

\section{SUPPLEMENTARY MATERIAL}

The Supplementary Material for this article can be found online at: https://www.frontiersin.org/articles/10.3389/fonc.2021. 781162/full\#supplementary-material

13. Tosi A, Cappellesso R, Dei Tos AP, Rossi V, Aliberti C, Pigozzo J, et al. The Immune Cell Landscape of Metastatic Uveal Melanoma Correlates With Overall Survival. J Exp Clin Cancer Res (2021) 40:154. doi: 10.1186/s13046-021-01947-1

14. Robert C, Long GV, Brady B, Dutriaux C, Maio M, Mortier L, et al. Nivolumab in Previously Untreated Melanoma Without BRAF Mutation. $N$ Engl J Med (2015) 372:320-30. doi: 10.1056/NEJMoa1412082

15. Larkin J, Chiarion-Sileni V, Gonzalez R, Grob JJ, Cowey CL, Lao CD, et al. Combined Nivolumab and Ipilimumab or Monotherapy in Untreated Melanoma. New Engl J Med (2015) 373:23-34. doi: 10.1056/NEJMoa1504030

16. Robert C, Schachter J, Long GV, Arance A, Grob JJ, Mortier L, et al. Pembrolizumab Versus Ipilimumab in Advanced Melanoma. N Engl J Med (2015) 372:2521-32. doi: 10.1056/NEJMoa1503093

17. Kirchberger MC, Moreira A, Erdmann M, Schuler G, Heinzerling L. Real World Experience in Low-Dose Ipilimumab in Combination With PD-1 Blockade in Advanced Melanoma Patients. Oncotarget (2018) 9:28903-9. doi: 10.18632/oncotarget.25627

18. Robert C, Schachter J, Long GV, Arance A, Grob JJ, Mortier L, et al. Pembrolizumab Versus Ipilimumab in Advanced Melanoma. $N$ Engl J Med (2015) 372:2521-32. doi: 10.1056/NEJMoa1503093

19. Heppt MV, Steeb T, Schlager JG, Rosumeck S, Dressler C, Ruzicka T, et al. Immune Checkpoint Blockade for Unresectable or Metastatic Uveal Melanoma: A Systematic Review. Cancer Treat Rev (2017) 60:44-52. doi: 10.1016/j.ctrv.2017.08.009

20. Lo CK, Mertz D, Loeb M. Newcastle-Ottawa Scale: Comparing Reviewers' to Authors' Assessments. BMC Med Res Methodol (2014) 14:45. doi: 10.1186/ 1471-2288-14-45

21. Higgins JP, Thompson SG, Deeks JJ, Altman DG. Measuring Inconsistency in Meta-Analyses. BMJ (2003) 327:557-60. doi: 10.1136/bmj.327.7414.557

22. HJ P. Cochrane Handbook for Systematic Reviews of Interventions. Version 5.1. 0 [Updated March 2011]. United Kingdom: The Cochrane Collaboration (2011). Available at: https://training.cochrane.org/handbook/archive/v5.1/.

23. Algazi AP, Tsai KK, Shoushtari AN, Munhoz RR, Eroglu Z, Piulats JM, et al Clinical Outcomes in Metastatic Uveal Melanoma Treated With PD-1 and PD-L1 Antibodies. Cancer (2016) 122(21):3344-53. doi: 10.1002/cncr.30258

24. Bol KF, Ellebaek E, Hoejberg L, Bagger MM, Larsen MS, Klausen TW, et al. Real-World Impact of Immune Checkpoint Inhibitors in Metastatic Uveal Melanoma. Cancers (Basel) (2019) 11(10). doi: 10.3390/cancers11101489

25. Del Vecchio M, Di Guardo L, Ascierto PA, Grimaldi AM, Sileni VC, Pigozzo J, et al. Efficacy and Safety of Ipilimumab $3 \mathrm{mg} / \mathrm{Kg}$ in Patients With Pretreated, 
Metastatic, Mucosal Melanoma. Eur J Cancer (Oxford Engl 1990) (2014) 50:121-7. doi: 10.1016/j.ejca.2013.09.007

26. Heppt MV, Amaral T, Kahler KC, Heinzerling L, Hassel JC, Meissner M, et al. Combined Immune Checkpoint Blockade for Metastatic Uveal Melanoma: A Retrospective, Multi-Center Study. J Immunother Cancer (2019) 7(1):299. doi: 10.1186/s40425-019-0800-0

27. Heppt MV, Heinzerling L, Kahler KC, Forschner A, Kirchberger MC, Loquai C, et al. Prognostic Factors and Outcomes in Metastatic Uveal Melanoma Treated With Programmed Cell Death-1 or Combined PD-1/Cytotoxic TLymphocyte Antigen-4 Inhibition. Eur J Cancer (Oxford Engl 1990) (2017) 82:56-65. doi: 10.1016/j.ejca.2017.05.038

28. Joshua AM, Monzon JG, Mihalcioiu C, Hogg D, Smylie M, Cheng T. A Phase 2 Study of Tremelimumab in Patients With Advanced Uveal Melanoma. Melanoma Res (2015) 25(4):342-7. doi: 10.1097/CMR.0000000000000175

29. Kelderman S, van der Kooij MK, van den Eertwegh AJ, Soetekouw PM, Jansen RL, van den Brom RR, et al. Ipilimumab in Pretreated Metastastic Uveal Melanoma Patients. Results of the Dutch Working Group on Immunotherapy of Oncology (WIN-O). Acta Oncol (2013) 52(8):1786-8. doi: 10.3109/ 0284186X.2013.786839

30. Maio M, Danielli R, Chiarion-Sileni V, Pigozzo J, Parmiani G, Ridolfi R, et al. Efficacy and Safety of Ipilimumab in Patients With Pre-Treated, Uveal Melanoma. Ann Oncol (2013) 24(11):2911-5. doi: 10.1093/annonc/mdt376

31. Najjar YG, Navrazhina K, Ding F, Bhatia R, Tsai K, Abbate K, et al. Ipilimumab Plus Nivolumab for Patients With Metastatic Uveal Melanoma: A Multicenter, Retrospective Study. J Immunother Cancer (2020) 8(1). doi: 10.1136/jitc-2019-000331

32. Pelster MS, Gruschkus SK, Bassett R, Gombos DS, Shephard M, Posada L, et al. Nivolumab and Ipilimumab in Metastatic Uveal Melanoma: Results From a Single-Arm Phase II Study. J Clin Oncol (2021) 39(6):599-607. doi: $10.1200 /$ JCO.20.00605

33. Piulats JM, Espinosa E, de la Cruz Merino L, Varela M, Alonso Carrion L, Martin-Algarra S, et al. Nivolumab Plus Ipilimumab for Treatment-Naive Metastatic Uveal Melanoma: An Open-Label, Multicenter, Phase II Trial by the Spanish Multidisciplinary Melanoma Group (GEM-1402). J Clin Oncol (2021) 39(6):586-98. doi: 10.1200/JCO.20.00550

34. Rossi E, Pagliara MM, Orteschi D, Dosa T, Sammarco MG, Caputo CG, et al. Pembrolizumab as First-Line Treatment for Metastatic Uveal Melanoma. Cancer Immunol Immunother (2019) 68(7):1179-85. doi: 10.1007/s00262-019-02352-6
35. Rozeman EA, Prevoo W, Meier MAJ, Sikorska K, Van TM, van de Wiel BA, et al. Phase Ib/II Trial Testing Combined Radiofrequency Ablation and Ipilimumab in Uveal Melanoma (SECIRA-Um). Melanoma Res (2020) 30 (3):252-60. doi: 10.1097/CMR.0000000000000653

36. Sander MS, Stukalin I, Vallerand IA, Goutam S, Ewanchuk BW, Meyers DE, et al. Evaluation of the Modified Immune Prognostic Index to Prognosticate Outcomes in Metastatic Uveal Melanoma Patients Treated With Immune Checkpoint Inhibitors. Cancer Med (2021) 10:2618-26. doi: 10.1002/ cam4.3784

37. van der Kooij MK, Joosse A, Speetjens FM, Hospers GA, Bisschop C, de Groot JW, et al. Anti-PD1 Treatment in Metastatic Uveal Melanoma in the Netherlands. Acta Oncol (2017) 56(1):101-3. doi: 10.1080/ 0284186X.2016.1260773

38. Zimmer L, Vaubel J, Mohr P, Hauschild A, Utikal J, Simon J, et al. Phase II DeCOG-Study of Ipilimumab in Pretreated and Treatment-Naive Patients With Metastatic Uveal Melanoma. PloS One (2015) 10(3):e0118564. doi: 10.1371/journal.pone.0118564

39. Najjar YG, Navrazhina K, Ding F, Bhatia R, Tsai K, Abbate K, et al. Ipilimumab Plus Nivolumab for Patients With Metastatic Uveal Melanoma: A Multicenter, Retrospective Study. J Immunother Cancer (2020) 8:e000331. doi: $10.1136 /$ jitc-2019-000331

Conflict of Interest: The authors declare that the research was conducted in the absence of any commercial or financial relationships that could be construed as a potential conflict of interest.

Publisher's Note: All claims expressed in this article are solely those of the authors and do not necessarily represent those of their affiliated organizations, or those of the publisher, the editors and the reviewers. Any product that may be evaluated in this article, or claim that may be made by its manufacturer, is not guaranteed or endorsed by the publisher.

Copyright $\odot 2021$ Zhao, Xia, Zhang, Zou, Zhu and Zhang. This is an open-access article distributed under the terms of the Creative Commons Attribution License (CC BY). The use, distribution or reproduction in other forums is permitted, provided the original author(s) and the copyright owner(s) are credited and that the original publication in this journal is cited, in accordance with accepted academic practice. No use, distribution or reproduction is permitted which does not comply with these terms. 